\title{
A 65-Year-Old Female With an Endobronchial Mass Lesion
}

\author{
Anup Kumar Singh MD, Purvesh Patel MD, Frank Breuer MD, and Arunabh Talwar MD
}

\begin{abstract}
Glomus tumor is a rare, predominantly benign, soft tissue tumor. The lower respiratory tract is an uncommon site of origin of glomus tumor, so endobronchial glomus tumor is extremely rare. Such tumors are mostly benign and identified incidentally on imaging. Diagnosis is confirmed by immunohistochemical staining, and resection is the treatment of choice. We report a middle-age female with endobronchial glomus tumor. This is $\mathbf{2 3 r d}$ case of reported pulmonary glomus tumor, to the best of our knowledge. Key words: benign; glomus tumor; endobronchial tumor; soft tissue tumor; electrocautery. [Respir Care 2013;58(8):e95-e98. (c) 2013 Daedalus Enterprises]
\end{abstract}

\section{Introduction}

Endobronchial mass lesion may be an incidental finding of chest imaging or it may present with symptoms of dyspnea, cough, or hemoptysis. We present a case of an endobronchial mass lesion in a patient with renal cell carcinoma and chronic lymphocytic leukemia, which was incidentally detected on chest imaging. We will discuss the differential diagnosis of endobronchial lesions in this case review.

\section{Case Report}

A 65-year-old female presented to clinic with a complaint of non-resolving dry cough of 2 months duration. There was no history of fevers, chills, rigors, chest pain, dyspnea, or hemoptysis. Her medical history was notable for chronic lymphocytic leukemia, renal cell carcinoma, hypertension, schizophrenia, and depression. She had a

Dr Singh is affiliated with the Department of Internal Medicine, Unity Health System, Rochester, New York. Dr Patel and Dr Talwar are affiliated with the Department of Pulmonary Medicine and Critical Care, and Dr Breuer is affiliated with the Department of Pathology, North Shore University, Manhasset, New York.

The authors have disclosed no conflicts of interest.

Correspondence: Anup Kumar Singh MD, Department of Internal Medicine, Unity Health System, 1555 Long Pond Road, Rochester NY 14626. E-mail: rajput.anup@gmail.com.

DOI: $10.4187 /$ respcare. 01952 history of 30 pack-years of smoking, but no alcohol or drug use. The rest of the review of systems was normal.

On examination she was alert, awake, and oriented. Pulse 70 beats/min, blood pressure 120/80 $\mathrm{mm}$ of $\mathrm{Hg}$, saturation $98 \%$ on room air, weight $93 \mathrm{~kg}$. General physical examination was non-contributory with no thyromegaly, jugular venous distension, or pedal edema. Cardiac examination revealed soft $\mathrm{S} 1$ and loud $\mathrm{S} 2$, with no murmur. Chest examination showed decreased air entry at the bases. Abdominal and neurological examination was normal.

Routine laboratory tests were within normal limits except for mild anemia and hypercalcemia. The hypercalcemia resolved after resection of renal cell carcinoma. Electrocardiogram and pulmonary function tests were within normal limits.

Chest x-ray did not reveal any pulmonary pathology. Computed tomography (CT) scan showed a right lower lobe endobronchial mass lesion, $8 \mathrm{~mm}$ in size (Fig. 1). No other pulmonary nodules, masses, or areas of consolidation were identified. CT scan of the abdomen and pelvis revealed $6.8 \mathrm{~cm}$ solid, left upper pole renal neoplasm, and liver hemangiomas.

Bronchoscopy confirmed a large, vascular endobronchial lesion emanating from the posterior wall of the right lower lobe bronchus, which obstructed it almost completely (Fig. 2). A curvilinear endobronchial ultrasound probe (BF-UC160F-OL8, Olympus, Tokyo, Japan) was used to evaluate the vascularity of the tumor so that it could be resected safely. Endobronchial ultrasound revealed a highly vascular lesion. Electrocautery (up to 20 Watts) via snare loop ${ }^{1}$ was used to resect the mass. Most of the mass was resected, but remnants were present in the bronchus. At the end of the procedure the bronchoscope was able to 


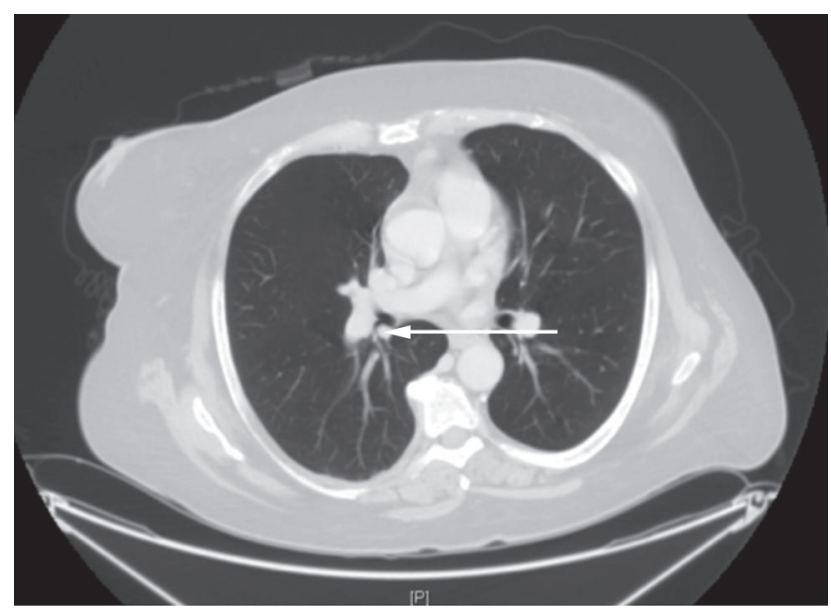

Fig. 1. Computed tomogram shows an 8-mm endobronchial mass in the right lower lobe.

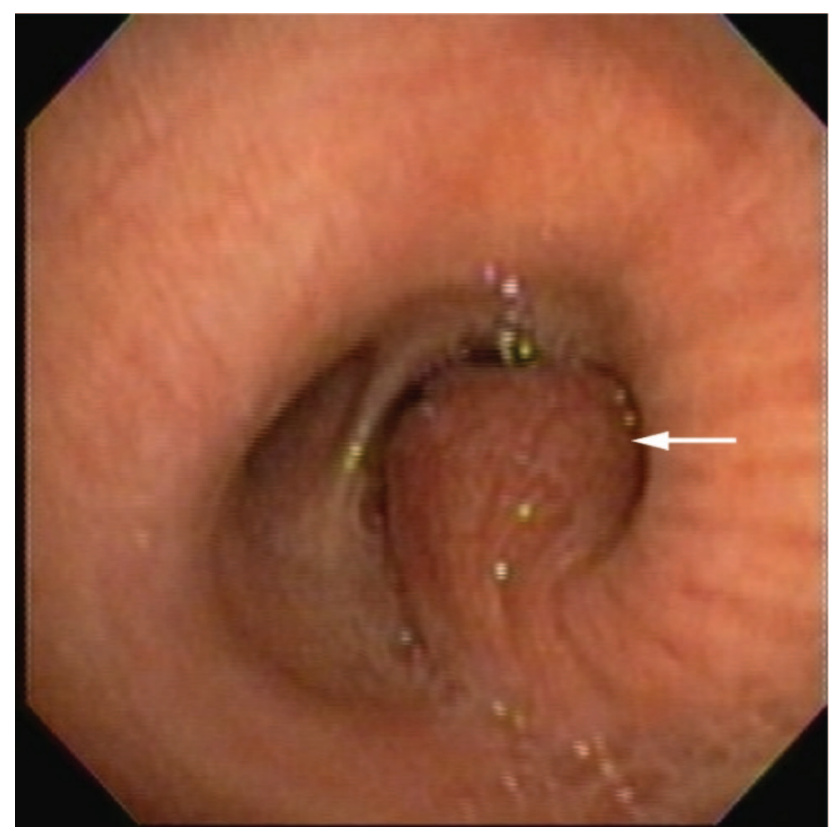

Fig. 2. Bronchoscopy shows the endobronchial mass obstructing the right lower bronchus.

pass through the lower bronchus to visualize the basilar segments, which were clear and normal in appearance.

The resected lesion was a $1.2 \times 0.4 \times 0.5 \mathrm{~cm}$ aggregate of red soft tan tissue. Microscopically, it was found to be composed of small rounded cells with prominent eosinophilic cytoplasm centering on and clustering around and in between gaping vascular spaces. No mitotic activity, cellular atypia, or necrosis was noted (Fig. 3). Immunohistochemical stains revealed positive staining of neoplastic cells for smooth-muscle actin and H-caldesmon, and negative staining for thyroid transcription factor 1 (TTF1), chromogranin, and synaptophysin. Immunohistochemical stain for Ki67 (MIB-1) revealed a proliferation index of

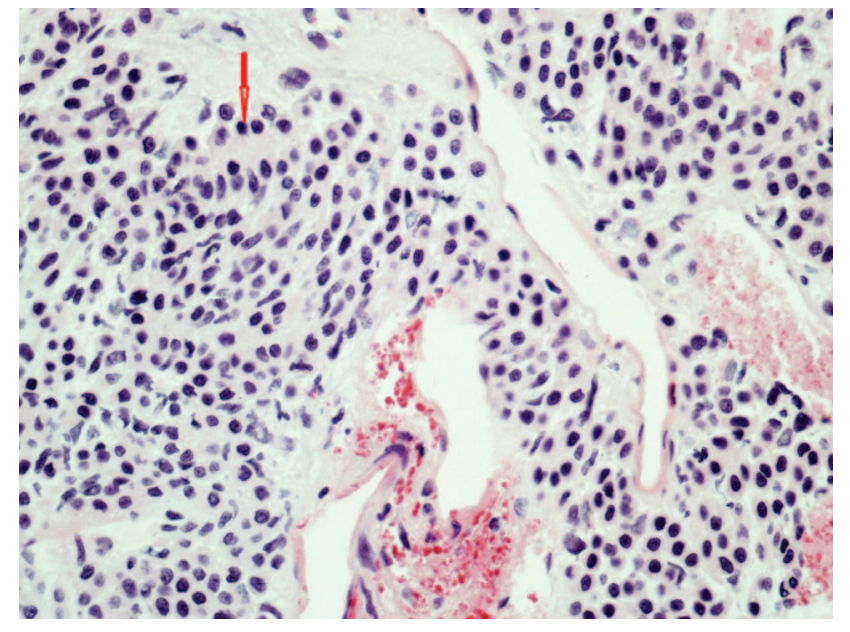

Fig. 3. Histology shows small rounded cells with prominent eosinophilic cytoplasm centering on and clustering around and between gaping vascular spaces. There is no mitotic activity, cellular atypia, or necrosis.

$<1 \%$. It was finally diagnosed as a benign solid glomus tumor.

\section{Discussion}

Glomus tumors are rare neoplasms derived from glomus bodies of dermis and subcutaneous tissues. They account for $1.6 \%$ of all soft tissue tumors, and can be present in almost all parts of body. Nail bed is the most common site for glomus tumor, and extremities account for $70 \%$ of all cases. $^{2}$ Glomus tumors are extremely rare in the lower respiratory tract, accounting for around 22 cases in the literature. Intrapulmonary glomus tumors are mostly benign, and only 4 cases of pulmonary malignant glomus tumor have been described. ${ }^{3}$ Generally, glomus tumors affect young adults of 20-30 years of age, with no sex predilection. However, glomus tumors specific to the lungs affect men twice as often as women, with an average age of around 48 years. ${ }^{3}$

Pulmonary glomus tumors are mostly asymptomatic and are usually detected accidentally on chest imaging. The size of most of the nodules in reported cases varies from 1 to $5 \mathrm{~cm}^{3}$. Chest x-ray may not detect very small parenchymal or endobronchial lesions, and appears normal in those cases. Chest $\mathrm{x}$-ray and CT scan findings may vary from solitary pulmonary nodules to a partial or complete collapse of lung segments due to endobronchial lesion. On CT scan a solitary pulmonary nodule appears as a well delineated round mass, which can distinguish it from irregular lung cancer lesions. Dynamic contrast-enhanced magnetic resonance imaging shows strong homogenous enhancement of glomus tumor due to its vascularity, and can distinguish these lesions from hamartomas, which show 


\section{A 65-Year-Old Female With an Endobronchial Mass Lesion}

Table. Immunohistochemistry for Differentiating Tumors Similar to Glomus Tumor

\begin{tabular}{|c|c|c|c|c|c|c|c|c|c|}
\hline Diagnosis & $\begin{array}{l}\text { Smooth } \\
\text { Muscle } \\
\text { Actin }\end{array}$ & Desmin & CD34 & Cytokeratin & $\begin{array}{l}\text { Neuron } \\
\text { Specific } \\
\text { Enolase }\end{array}$ & Chromogranin & S100 & CD99 & $\begin{array}{c}\text { Type } 4 \\
\text { Collagen }\end{array}$ \\
\hline Glomus tumor & + & \pm & \pm & - & - & - & - & - & + \\
\hline Hemangiopericytoma & - & - & + & - & - & - & - & - & \pm \\
\hline Carcinoid & - & - & - & + & + & + & - & - & - \\
\hline Paraganglioma & - & - & - & - & + & + & $+*$ & - & - \\
\hline Primitive neuroectodermal tumor & - & - & - & - & + & \pm & \pm & + & - \\
\hline
\end{tabular}

a lesser degree of contrast enhancement. ${ }^{4}$ Glomus tumor should be kept as a differential diagnosis in case of round masses showing strong enhancement on pulmonary magnetic resonance imaging. Despite all the advances, imaging studies cannot distinguish pulmonary glomus tumor from carcinoid tumor, hemangioma, or metastatic tumor.

Benign glomus tumors can be divided pathologically into 3 subtypes: glomus tumor proper, glomangioma, and glomangiomyoma. Glomus tumor proper has a predominance of round glomus cells, as seen in this case, while glomangioma and glomangiomyoma have a predominance of blood vessels and spindle cells, respectively. Glomus tumor proper is the most common of the 3 , followed by glomangioma and then glomangiomyoma, the rarest of all, with a frequency of about $8 \% .^{2}$ Malignant and atypical glomus tumors of the lung are very rare. These can be classified as malignant glomus tumor (glomangiosarcoma), glomus tumor with nuclear atypia only (symplastic glomus tumor), glomus tumor of uncertain malignant potential, and glomangiomatosis (histologically benign glomus tumor with diffuse growth). ${ }^{5}$

Glomus tumors have a wide range of differential diagnoses, including carcinoid tumor, smooth-muscle neoplasm, paraganglioma, primitive neuroectodermal tumor, metastatic tumor, and hemangiopericytoma. A uniform cell with central nuclei and clear to eosinophilic cytoplasm on histology suggests the differential diagnosis of glomus tumor, carcinoid tumor, paraganglimona, and, occasionally, primitive neuroectodermal tumor. Diagnosis can only be confirmed on immunohistochemical staining (Table). ${ }^{6}$

Glomus tumors are most often confused with carcinoid tumor, due to similar histology. Immunohistology is important for differentiating glomus tumor from carcinoid tumor. Glomus tumors are positive for smooth-muscle actin and type 4 collagen, and are negative for neuroendocrine markers of carcinoid tumor, such as synaptophysin, chromogranin A, and cytokeratin. ${ }^{6}$ Immunostaining in our case was strongly positive for smooth-muscle actin and
$\mathrm{H}$-caldesmon (markers of smooth-muscle neoplasm), and negative for cytokeratin and neuroendocrine markers.

Hemangiopericytoma shows branching vessels (staghorntype vessels) in close proximity to tumor cells, and is similar to glomus tumor. Microscopically, hemangiopericytoma does not have epithelioid cells with round central nuclei, like glomus tumor, but is composed of spindle cells with elongated nuclei. Hemangiopericytoma is negative for smooth-muscle actin and positive for CD34 and CD57.7

Smooth-muscle neoplasm should also be considered in the differential diagnosis of glomus tumor, where an immunohistochemical profile is less important in diagnosis than the histological appearance. Leiomyoma of the respiratory tract is an unusual tumor that involves either bronchus or alveolar parenchyma. It expresses smooth-muscle actin and desmin, and shows fascicles of mature, spindled, smooth-muscle cells unlikely to be confused with the round or polygonal cells of glomus tumor. ${ }^{6}$

Glomus tumors of lungs are mostly benign, and complete surgical resection is the treatment of choice. Glomus tumor confined to the lumen of the bronchus, as in our case, can be resected by bronchoscopic techniques like neodymium-yttrium-aluminum-garnet laser, electrocoagulation, forceps extraction, and cryotherapy. ${ }^{8}$ If the tumor extends through the tracheobronchial wall, or if a small tumor involves the parenchyma, limited surgical resection (sleeve and wedge parenchyma-sparing bronchial resection) would be the treatment of choice. For malignant and large parenchymal glomus tumors, sublobar resection and lobectomy are the treatment of choice. ${ }^{9,10}$ Even with metastases, patients have an excellent prognosis.

Glomus tumors are rare, and few cases of endobronchial glomus tumor have been described in the literature. To the best of our knowledge, this is only the 23rd case of lower respiratory tract glomus tumor to be reported. Awareness about glomus tumors as differential diagnoses of endobronchial vascular lesion is important to clinicians for effective management of these cases. 


\section{A 65-Year-Old Female With an Endobronchial Mass Lesion}

\section{REFERENCES}

1. Kawahara M, Furuse K, Kodama N, Ogawara M, Atagi S, Kamimori T, Nakao M, Naka N. Endobronchial electrocautery using snare. Diagn Ther Endosc 1996;2(4):207-210.

2. Weiss S, Goldblum J. Perivascular tumors. In: Soft tissue tumors, 5 th edition. St Louis: Mosby/Elsevier; 2008:751-767.

3. De Cocker J, Messaoudi N, Waelput W, Van Schil PE. Intrapulmonary glomus tumor in a young woman. Interact Cardiovasc Thorac Surg 2008;7(6):1191-1193

4. Ueno M, Nakashima O, Mishima M, Yamada M, Kikuno M, Nasu K, Kudo S. Pulmonary glomus tumor: CT and MRI findings. J Thorac Imaging 2004;19(2):131-134.

5. Folpe AL, Fanfurg-Smith JC, Miettinen M, Weiss SW. Atypical and malignant glomus tumors: analysis of 53 cases with a proposal for the reclassification of glomus tumors. Am J Surg Pathol 2001;25(1): $1-12$.
6. Gaertner EM, Steinberg DM, Huber M, Hayashi T, Tsuda N, Askin FB, et al. Pulmonary and mediastinal glomus tumors-report of five cases including a pulmonary glomangiosarcoma: a clinicopathologic study with literature review. Am J Surg Pathol 2000;24(8):1105-1114.

7. Porter PL, Bigler SA, McNutt M, Gown AM. The immunophenotype of hemangiopericytomas and glomus tumors, with special reference to muscle protein expression: an immunohistochemical study and review of the literature. Mod Pathol 1991;4(1):46-52.

8. Vailati P, Bigliazzi C, Casoni G, Gurioli C, Saragoni L, Poletti V. Endoscopic removal of a right main bronchus glomus tumor. Monaldi Arch Chest Dis 2004;61(2):117-119.

9. Takahashi N, Oizumi H, Yanagawa N, Sadahiro M. A bronchial glomus tumor surgically treated with segmental resection. Interact Cardiovasc Thorac Surg 2006;5(3):258-260.

10. Lucchi M, Melfi F, Ribechini A, Dini P, Duranti L, Fontanini G, Mussi A. Sleeve and wedge parenchyma-sparing bronchial resections in low-grade neoplasms of the bronchial airway. J Thorac Cardiovasc Surg 2007;134(2):373-377. 